\title{
THE n-FOLD CONVOLUTION OF A MIXED DENSITY AND MASS FUNCTION*,**
}

\author{
Christoph Haehling von Lanzenauer *** \\ AND \\ WILLIAM N. LUNDBERG ***
}

\begin{abstract}
The distribution of the sum of $n$ mutually independent random variables with a common distribution $f(x)$ plays an important role in many insurance problems. The paper presents alternative methods of deriving the distribution of the sum of $n$ random variables when $f(x)$ is a mixed density and mass function. The methods are illustrated and compared.
\end{abstract}

\section{INTRODUCTION}

The sum of $n$ mutually independent random variables $x_{i}$ plays an important role in many insurance problems. Of particular interest however, is the case where the $n$ mutually independent random variables have a common piece-wise continuous density function $f\left(x_{i}\right)$. The density function of the sum $X_{n}=x_{1}+\ldots+x_{n}$ is the $n$-fold convolution of $f\left(x_{i}\right)$ with itself and is denoted by $f^{n *}\left(X_{n}\right)$ [3]. There exist however situations where the $n$ random variables have a common mixed density and mass function. Typical examples relate to insurance contracts with a provision for deductibles and/or insurance limits $[4,8]$. The problem arises also in the derivation of the distribution of aggregate claims when a pooling limit is applied to each certificate [7]. The density of the sum of the $n$ random variables is then the $n$-fold convolution of the mixed density and mass function with itself. Clearly, for large values of $n$ this density

* This paper has been supported by a grant from the Richard Ivey Foundation.

** The authors wish to acknowledge the helpful comments and suggestions made by Dr. P. A. Fraser, Department of Applied Mathematics, University of Western Ontario.

*** The authors are Associate Professor and Research Assistant respectively in the School of Business Administration, University of Western Ontario. 
can be approximated by the normal distribution. For smaller values of $n$, however, the density function must be derived in a different way.

The purpose of this paper is to present alternative methods of deriving the density function of the sum of $n$ independent random variables with a common mixed density and mass function.

\section{Formulation of Alternative Methods}

In this section, three methods are described which may be used to derive the $n$-fold convolution of a mixed density and mass function. The methods are referred to as

I) Analytic Method

2) Numeric Method

3) Method of Moments.

In order to illustrate the methods, the following mixed density and mass function has been chosen ${ }^{1}$ ).

$$
f(x)= \begin{cases}\lambda e^{-\lambda x}+A \cdot \delta(x-D) & \text { for } 0 \leqslant x \leqslant D \\ 0 & \text { otherwise }\end{cases}
$$

where

$$
A=\int_{D^{+}}^{\infty} \lambda e^{-\lambda x} d x=e^{-\lambda D}
$$

and

$\delta(x-D)= \begin{cases}\lim _{\Delta x \rightarrow 0}(\mathrm{I} / \Delta X) & \text { for } D-(\Delta x / 2) \leqslant x \leqslant D+(\Delta x / 2) \\ 0 & \text { otherwise. }\end{cases}$

$\delta(x-D)$, known as the Dirac delta function, may be thought of as the height of a unit impulse, centered on $x=D$, as the width tends to zero ${ }^{2}$ ).

1) This situation represents the distribution of the retention cost $x$ for a given loss to the insurance consumer with an insurance policy having a deductible $D$. Under such a deductible arrangement the insured has to absorb the entire loss if the loss is less than or equal to $\mathrm{D}$ and has to carry an amount equal to $D$ if the loss exceeds $D$.

2) For discussion of the Dirac delta function, see Lighthill, M. J., Introduction to Fourier Analysis and Generalized Functions, Cambridge University Press, I958 [5]. 
Figure I illustrates the function.

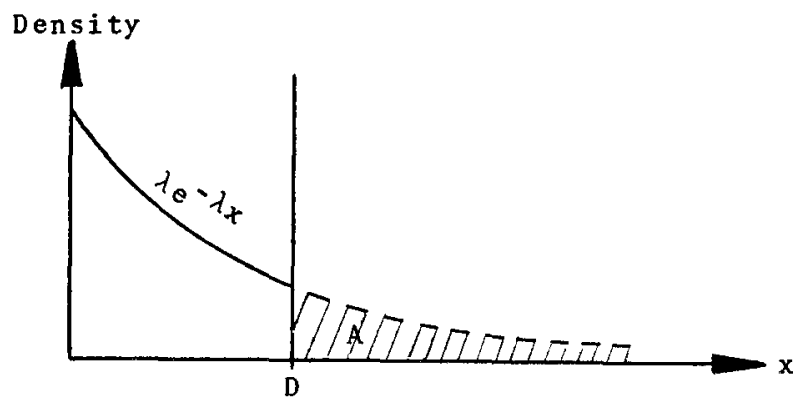

Figure I

I. Analytic Method

The analytic method is based on Laplace Transform theory [I] which states

$$
L\left[\int_{0}^{z} g(x) h(Z-x)\right] d x=L[g(x)\rceil \cdot L[h(y)]
$$

with $g(x)=0$ for $x<0$ and $h(y)=0$ for $y<0$.

For the special case of the sum, $X_{n}$, of $n$ random variables $x$ with an identical distribution $f(x)$ the density function of $X_{n}$ is the $n$-fold convolution of $f(x)$ with itself and is denoted by $f^{n *}\left(X_{n}\right)$ [2]. Thus

$$
L\left[f^{n *}\left(X_{n}\right)=\{L[f(x)]\}^{n} .\right.
$$

For a piece-wise continuous function, (4) may be rewritten as

$$
L\left[f^{n *}\left(X_{n}\right)\right]=\left\{\int_{0}^{\infty} f(x) e^{-s x} d x\right\}^{n} .
$$

Inversion of the right hand side of (5) results in the desired $f^{n *}\left(X_{n}\right)$.

\section{Illustration}

The above concept will now be applied to the mixed density and mass function described by (2).

The Laplace Transform of (2) is given by (6)

$$
\begin{gathered}
L[f(x)]=\int_{0}^{D} \lambda e^{-\lambda x} e^{-s x} d x+\int_{D}^{\infty} 0 \cdot e^{-s x} d x+\int_{0}^{\infty} A \delta(x-D) e^{-s x} d x \\
=\frac{\lambda}{s+\lambda}-\frac{\lambda}{s+\lambda} e^{-(s+\lambda) D}+A e^{-s D} .
\end{gathered}
$$


Raising (6) to the power of $n$ results in (7).

$L\left[f^{n *}\left(X_{n}\right)\right]=\sum_{k=0}^{n} \sum_{i=0}^{k}(-\mathrm{I})^{i}\left(\begin{array}{l}n \\ k\end{array}\right)\left(\begin{array}{l}k \\ i\end{array}\right) A^{k-i} \lambda^{n-k+i}\left[e^{-\lambda_{i} D} e^{-k s D}(\mathrm{I} / s+\lambda)^{n-k+i}\right]$.

The inversion of the right hand side of (7) results in (8).

$$
\begin{aligned}
& f^{n *}\left(X_{n}\right)=\sum_{k=0}^{n} \sum_{i=0}^{k}(-\mathrm{I})^{i} \frac{n !}{(n-k) !(k-i) ! i !} \lambda^{n-k+i} A^{k-i} \\
& \left\{\begin{array}{c}
\delta_{r k} \delta\left(X_{n}-k D\right)+ \\
\quad+\left(\mathrm{I}-\delta_{r k}\right) U\left(X_{n}-k D\right) \frac{\left(X_{n}-k D\right)^{n-k+i-1} e^{-\lambda\left[X_{n}-D(k-i)\right]}}{(n-k+i-\mathrm{I}) !} \\
\quad \text { for } 0 \leqslant X_{n} \leqslant n D
\end{array}\right\}
\end{aligned}
$$

where $r=n+i$

$$
\begin{aligned}
\delta_{r k}=\left\{\begin{array}{l}
\mathrm{I} \text { for } r=k \\
0 \text { for } r \neq k
\end{array}\right. \\
U\left(X_{n}-k D\right)=\left\{\begin{array}{l}
0 \text { for }-\infty \leqslant X_{n}<k D \\
\mathrm{I} / 2 \text { for } X_{n}=k D \\
\mathrm{I} \text { for } k D<X_{n} \leqslant \infty
\end{array} \quad\right. \text { [UNIT STEP] }
\end{aligned}
$$

Thus (8) represents the desired $f^{n *}\left(X_{n}\right)^{3}$ )

\section{Numeric Method}

Using the numeric method, the domain of the random variable $x$ is divided into $M+\mathrm{I}$ discrete intervals. Let $a$ and $b$ represent the lower and upper limit of the domain. A discrete probability dis-

3) It is interesting to note that for $D \rightarrow \infty$ (i.e. exponential distribution) the only term that contributes to the convolution in the finite region is the term with $k=i=0$. Thus for this case, (8) reduces to

$$
f^{n *}\left(X^{n}\right)=\frac{\lambda^{n} X_{n}^{n-1}}{(n-\mathrm{I}) !} e^{-\lambda X_{n}}=\frac{\lambda\left(\lambda X_{n}\right)^{n-1}}{\Gamma(n-\mathrm{I}+)} e^{-\lambda X_{n}}
$$

which is a gamma distribution. This finding is consistant with the results given in [9]. 
tribution for $x$ and $X_{n}$ may be found using the following relationships $\left.{ }^{4}\right)$

$$
\begin{aligned}
& P\left(a+\frac{(b-a) j}{M}\right)=P^{1 *}\left(a+\frac{(b-a) j}{M}\right)= \\
& \quad=\left\{\begin{array}{c}
\int_{a}^{a+\frac{b-a}{2 M}} f(x) d x \quad \text { for } j=0 \\
a+\frac{(b-a)(2 j+1)}{\int^{2 M}} f(x) d x \text { for } j=\mathrm{I}, 2, \alpha \ldots M-\mathrm{I} \\
a+\frac{(b-a)(2 j-1)}{2 M}
\end{array}\right. \\
& P^{n *}\left(n a+\frac{\int_{b-\frac{b-a}{2 M}}^{b} f(x) d x \quad \text { for } j=M}{M(b-a)}\right)=\sum_{j=\alpha}^{\beta} P^{(n-1) *}\left(\left(n a+\frac{m(b-a)}{M}\right)-a-\right. \\
& \left.\quad-\frac{j(b-a)}{M}\right) \cdot P\left(a+\frac{j(b-a)}{M}\right)=\sum_{i=\alpha}^{\beta} P^{(n-1) *}((n-\mathrm{I}) a+ \\
& \left.\quad \text { for } m=\frac{(m-j)(b-a)}{M}\right) \cdot P\left(a+\frac{j(b-a)}{M}\right)
\end{aligned}
$$

$$
\text { where } \alpha= \begin{cases}\text { for } m \leqslant(n-\mathrm{I}) M \\ m-(n-\mathrm{I}) M & \text { for } m>(n-\mathrm{I}) M \\ & \text { and } \beta= \begin{cases}m & \text { for } m \leqslant M \\ M & \text { for } m>M .\end{cases} \end{cases}
$$

\section{Illustration}

The above procedure is now applied to the mixed density and mass function described by (2) with $a=0$ and $b=D$. Thus, (9) and (ro) become

4) It is recognized that the handling of the first $(j=0)$ and the last $(j=M)$ interval in (9) is one of various possibilities. 


$$
\begin{aligned}
& P\left(\frac{j D}{M}\right)=P^{1 *}\left(\frac{j D}{M T}\right)=
\end{aligned}
$$

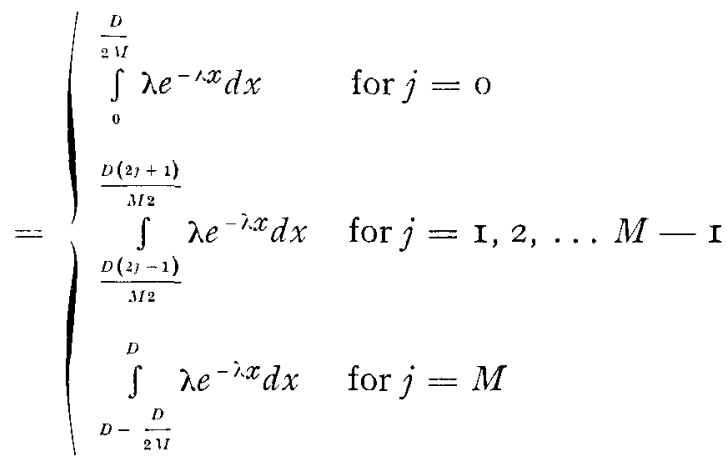

and $\quad P^{n *}\left(\frac{m D}{M}\right)=\sum_{i=\alpha}^{\beta} P^{(n-1) *}\left(\frac{(m-j) D}{M}\right) \cdot P\left(\frac{j D}{M}\right)$.

Clearly, $M$ plays an important role on how accurately the discrete approximation (I I) portrays the mixed density and mass function $f(x)$ as given in (2). To obtain an indication of the closeness one may compute the mean, standard deviation, moment coefficients of skewness and kurtosis for various values of $M$. Table $I$ represents these results for $\lambda=0.007$ and $D=$ Ioo.

TABLE I

\begin{tabular}{|c|c|c|c|c|}
\hline $\mathrm{m}$ & mean & Standard deviation & Skewness & Kurtosis \\
\hline I & $7^{0.4688}$ & 45.6183 & -0.8974 & I. 8053 \\
\hline 5 & 71.8577 & 34.9885 & $-0.7^{876}$ & 2.0877 \\
\hline Io & $71.9017^{\circ}$ & 34.5922 & -0.7638 & 2.0157 \\
\hline 25 & 71.9140 & $34.4^{803}$ & -0.7567 & I.993 I \\
\hline-50 & $7 \times .9 \times 5^{8}$ & $34 \cdot 4643$ & -0.7557 & I. .9898 \\
\hline IOO & 71.9162 & 34.4603 & -0.7554 & I.9889 \\
\hline $25^{\circ}$ & 71.9164 & 34.4592 & -0.7553 & I. 9887 \\
\hline 500 & 71.9164 & $34.459^{\circ}$ & -0.7553 & I. 9887 \\
\hline$\infty^{5}$ & $7 x .9164$ & 34.4590 & -0.7553 & 1.9887 \\
\hline
\end{tabular}

5 For $M=\infty$ the value of the descriptive measures may be obtained from the following expressions:

Mean $=\left\{\mathrm{I} / \lambda\left[\mathrm{I}-e^{-\lambda D}\right]\right\}$

Standard

Deviation $=\left\{\mathbf{I} / \lambda^{2}\left[\mathrm{I}-2 D \lambda e^{-\lambda D} e^{-2 \lambda D}\right]\right\}^{1 / 2}=\sigma$

Skewness $=\left\{\mathrm{I} / \lambda^{3}\left[2-3 D^{2} \lambda^{2} e^{-\lambda D}-6 D \lambda e^{-2 \lambda D}-2 e^{-3 \lambda D}\right]\right\} / \sigma^{3}$

Kurtosis $=\left\{\mathrm{I} / \lambda^{4}\left[9-\left(4 D^{3} \lambda^{3}+\mathrm{I} 2 D \lambda\right) e^{-\lambda D}-\left(\mathrm{I} 2 D^{2} \lambda^{2}+6\right) e^{-2 \lambda D}-\right.\right.$ - $\left.\left.\mathrm{I} 2 D \lambda e^{-3 \lambda} D-3 e^{-4 \lambda D}\right]\right\} \mathrm{T} / \sigma^{4}$ 
The descriptive measures of (II) should be compared to those of the mixed density and mass function (2) since some may be close while others deviate substantially. For example with a symmetric distribution it is conceivable for a given value of $M$ that the mean and skewness could be close while the standard deviation and kurtosis could deviate significantly.

As can be seen from Table $I$, all of the descriptive measures converge to those obtained for $M=\infty$ as $M$ increases $\left.{ }^{6}\right)$. On the other hand, as $M$ increases the number of calculations and storage requirements increase drastically. Thus the value of $M$ must be chosen in light of these trade-offs.

\section{Method of Moments}

With the method of moments an approximation to the $n$-fold convolution is obtained in a two step process. First the mean and the second, third and fourth moments about the mean for $f^{n *}\left(X_{n}\right)$ are determined. In the second step, a density function consistent with these descriptive measures is derived.

As shown in [3] the mean and the second moment about the mean of the sum of $n$ mutually independent random variables with a common distribution (with a finite mean of $\mu$ and a finite variance of $\left.\sigma^{2}\right), \mu_{n}$ and $\theta_{n}^{2}$, are

$$
\begin{aligned}
& \mu_{n}=n \mu \\
& \theta_{n}^{2}=\sigma_{n}^{2}=n \sigma^{2} .
\end{aligned}
$$

As shown in Appendix A, the third and fourth moments about the mean of the sum of $n$ mutually independent random variables with a common distribution, $\theta_{n}^{3}$ and $\theta_{n}^{4}$, are given by (I5) and (I6) respectively

$$
\begin{gathered}
\theta_{n}^{3}=n \theta^{3} \\
\theta_{n}^{4}=n \theta^{4}+3\left(n^{2}-n\right)\left(\sigma^{2}\right)^{2} .
\end{gathered}
$$

As a second step a density function has to be determined which is consistent with the descriptive measures calculated in (I3) through (I6). In light of the independence assumption, the normal distri-

6) The rate of convergence depends on $\lambda$ and $D$. 
bution seems to be a logical choice when $n$ is large $\left.{ }^{7}\right)$. In cases where the test for normality [2] fails, the beta distribution may be used successfully ${ }^{8}$.

\section{Illustration}

The four descriptive measures $\mu, \theta^{2}, \theta^{3}$ and $\theta^{4}$ for the function given by (2) can be determined by evaluating the expressions within the brackets \{\} in footnote 5 . Various examples of the resulting density function for $f^{n *}\left(X_{n}\right)$ using the beta distribution are shown in Figure 2.

\section{Comparison of Alternative Methods}

The usefulness of the alternative methods of deriving the distribution of the sum of $n$ independent random variables with a common mixed density and mass function in the context of (2) can best be analysed on a comparative basis.

\section{Analytic versus Numeric}

The density function derived by the analytic method has to be integrated to obtain a discrete probability distribution which then can be compared to that derived by the numeric method. Table 2 represents the results for $n=\lambda, 5=0.007$ and $D=I 00$. As can be observed from the errors, the accuracy of the numeric method improves significantly as $M$ increases from Io to 50. Thus, in the context of (2) there exists a trade-off between the achieved accuracy and the computational effort. For problems where a high degree of accuracy is necessary, the numeric method requires a large value of $M$ thereby making the analytic method the more efficient procedure.

7) Note that

$$
\begin{gathered}
\lim _{n \rightarrow \infty} \frac{n \theta^{3}}{\left(n \theta^{2}\right)^{3 / 2}}=0 \\
\lim _{n \rightarrow \infty} \frac{n \theta^{4}+3\left(n^{2}-n\right)}{\left(n \theta^{2}\right)^{2}}=3
\end{gathered}
$$

which are necessary but not sufficient conditions for normality.

$\left.{ }^{8}\right)$ A procedure for obtaining the beta distribution consistent with the four descriptive measures is outlined in [6]. 
TABLE 2

\begin{tabular}{|c|c|c|c|c|c|}
\hline \multirow{3}{*}{$\mathrm{X}$} & \multicolumn{3}{|c|}{ Probability } & \multicolumn{2}{|c|}{ Error } \\
\hline & \multirow[t]{2}{*}{ Analytic } & \multicolumn{2}{|c|}{ Numeric } & \multirow[b]{2}{*}{$\mathrm{M}=\mathrm{IO}$} & \multirow[b]{2}{*}{$M=5^{\circ}$} \\
\hline & & $\mathrm{M}=\mathbf{I 0}$ & $M=5^{\circ}$ & & \\
\hline $0-5$ & 0.000000 & 0.000000 & 0.000000 & 0.000000 & 0.000000 \\
\hline $5^{-}$I5 & 0.000000 & 0.000000 & 0.000000 & 0.000000 & 0.000000 \\
\hline$I_{5}-25$ & 0.000001 & 0.000002 & $0.00000 \mathrm{I}$ & -0.000001 & 0.000000 \\
\hline $25-35$ & 0.000005 & 0.000007 & 0.000005 & -0.000002 & 0.000000 \\
\hline $35-45$ & 0.000014 & 0.000017 & $0.00001_{4}$ & -0.000002 & 0.000000 \\
\hline $45^{-} 55$ & 0.000031 & 0.000036 & 0.000032 & -0.000005 & $-0.00000 \mathrm{I}$ \\
\hline $55-65$ & 0.000060 & 0.000066 & $0.00006 \mathrm{I}$ & -0.000005 & -0.000001 \\
\hline $65-75$ & 0.000104 & 0.0001 II & $0.00010_{4}$ & -0.000006 & 0.000000 \\
\hline $75-85$ & 0.000165 & 0.000173 & 0.000165 & -0.000008 & 0.000000 \\
\hline $85-95$ & 0.000246 & 0.000255 & 0.000246 & -0.000009 & 0.000000 \\
\hline $95-105$ & 0.000349 & 0.000363 & 0.000349 & -0.000013 & 0.000000 \\
\hline 105 -II5 & 0.000487 & 0.000513 & 0.000488 & -0.000025 & $-0.00000 \mathrm{I}$ \\
\hline II 5 -I 25 & 0.000698 & 0.000734 & 0.000700 & -0.000035 & -0.000002 \\
\hline $125-135$ & 0.001017 & 0.001060 & 0.001018 & -0.000043 & $-0.00000 \mathrm{I}$ \\
\hline I $35-145$ & $0.00 \times 462$ & 0.001509 & 0.001464 & -0.000046 & -0.000002 \\
\hline I $45^{-I} 55$ & 0.002045 & 0.002093 & 0.002047 & -0.000047 & -0.000002 \\
\hline I $55-165$ & 0.002765 & 0.002812 & 0.002767 & -0.000047 & -0.000002 \\
\hline $165-175$ & 0.003616 & $0.00366 I$ & 0.003618 & -0.000045 & -0.000002 \\
\hline I $75-185$ & 0.004589 & 0.004630 & 0.004590 & -0.000040 & -0.000000 \\
\hline $185-195$ & 0.005668 & 0.005705 & 0.005669 & -0.000037 & -0.000001 \\
\hline $195-205$ & 0.006853 & 0.006962 & 0.006858 & -0.000109 & -0.000005 \\
\hline $205-2 I 5$ & 0.008478 & 0.008625 & 0.008484 & -0.000146 & -0.000005 \\
\hline $215-225$ & 0.010725 & 0.010820 & 0.010729 & -0.000095 & -0.000004 \\
\hline $225-235$ & 0.013379 & 0.0I343I & $0.0133^{81}$ & -0.000052 & -0.000002 \\
\hline $235-245$ & $0.01625 \mathrm{I}$ & $0.01627 \mathrm{I}$ & 0.016252 & -0.000020 & $-0.00000 \mathrm{I}$ \\
\hline $245^{-255}$ & 0.019194 & 0.019186 & 0.019194 & 0.000008 & 0.000000 \\
\hline $255^{-265}$ & 0.022089 & $0.02206 \mathrm{I}$ & 0.022088 & 0.000027 & 0.000000 \\
\hline $265-275$ & $0.02484^{8}$ & 0.024805 & 0.024847 & 0.000043 & 0.000001 \\
\hline $275-285$ & 0.027404 & 0.027349 & 0.027402 & 0.000055 & 0.000002 \\
\hline $285-295$ & 0.029710 & 0.029646 & $0.0297^{\circ} 7$ & 0.000064 & 0.000002 \\
\hline $295-305$ & $0.03244^{\circ}$ & 0.032969 & $0.03246 \mathrm{I}$ & -0.000528 & -0.000020 \\
\hline $305-315$ & 0.038405 & $0.03^{80} 7^{\circ}$ & $0.03839 \mathrm{I}$ & 0.000335 & 0.000013 \\
\hline $315-325$ & 0.043 I 54 & 0.042877 & 0.043143 & 0.000276 & 0.000010 \\
\hline $325-335$ & 0.046237 & 0.046011 & 0.046228 & 0.000226 & 0.000009 \\
\hline $335-345$ & 0.047953 & 0.047769 & 0.047945 & 0.000183 & 0.000008 \\
\hline $345-355$ & 0.048555 & 0.048407 & 0.048549 & 0.000147 & 0.000005 \\
\hline $355-365$ & 0.048263 & 0.048146 & $0.04^{82} 5^{8}$ & 0.000 II 7 & 0.000004 \\
\hline $365-375$ & 0.047262 & $0.04717^{2}$ & 0.047259 & 0.000090 & 0.000003 \\
\hline $375-3^{8} 5$ & 0.045712 & 0.045644 & 0.045709 & 0.000068 & 0.000002 \\
\hline $385-395$ & 0.043746 & 0.043697 & 0.043744 & 0.000048 & $0.00000 \mathrm{I}$ \\
\hline $395-405$ & 0.051219 & 0.050534 & 0.051192 & 0.000684 & 0.000027 \\
\hline $405-4$ I 5 & 0.053628 & 0.053774 & 0.053634 & -0.000146 & -0.000005 \\
\hline $415-425$ & 0.045630 & 0.045756 & 0.045635 & -0.000126 & -0.000005 \\
\hline $425-435$ & 0.038687 & 0.038797 & $0.03869 \mathrm{I}$ & -0.000110 & -0.000004 \\
\hline $435-445$ & $0.03267^{2}$ & 0.032768 & 0.032676 & -0.000096 & -0.000004 \\
\hline $445-455$ & 0.027473 & $0.02755^{6}$ & 0.027476 & -0.000082 & -0.000002 \\
\hline $455-465$ & 0.022990 & 0.023062 & 0.022993 & $-0.00007 x$ & -0.000002 \\
\hline $465-475$ & 0.019133 & 0.019195 & 0.019136 & -0.000062 & -0.000002 \\
\hline $475-4^{85}$ & $0.015^{825}$ & 0.015878 & 0.015827 & -0.000052 & $-0.00000 \mathrm{I}$ \\
\hline $4^{8} 5-495$ & 0.012996 & 0.013042 & 0.012998 & -0.000045 & -0.000002 \\
\hline $495-500$ & 0.035767 & 0.035973 & 0.035775 & -0.000206 & -0.000008 \\
\hline
\end{tabular}




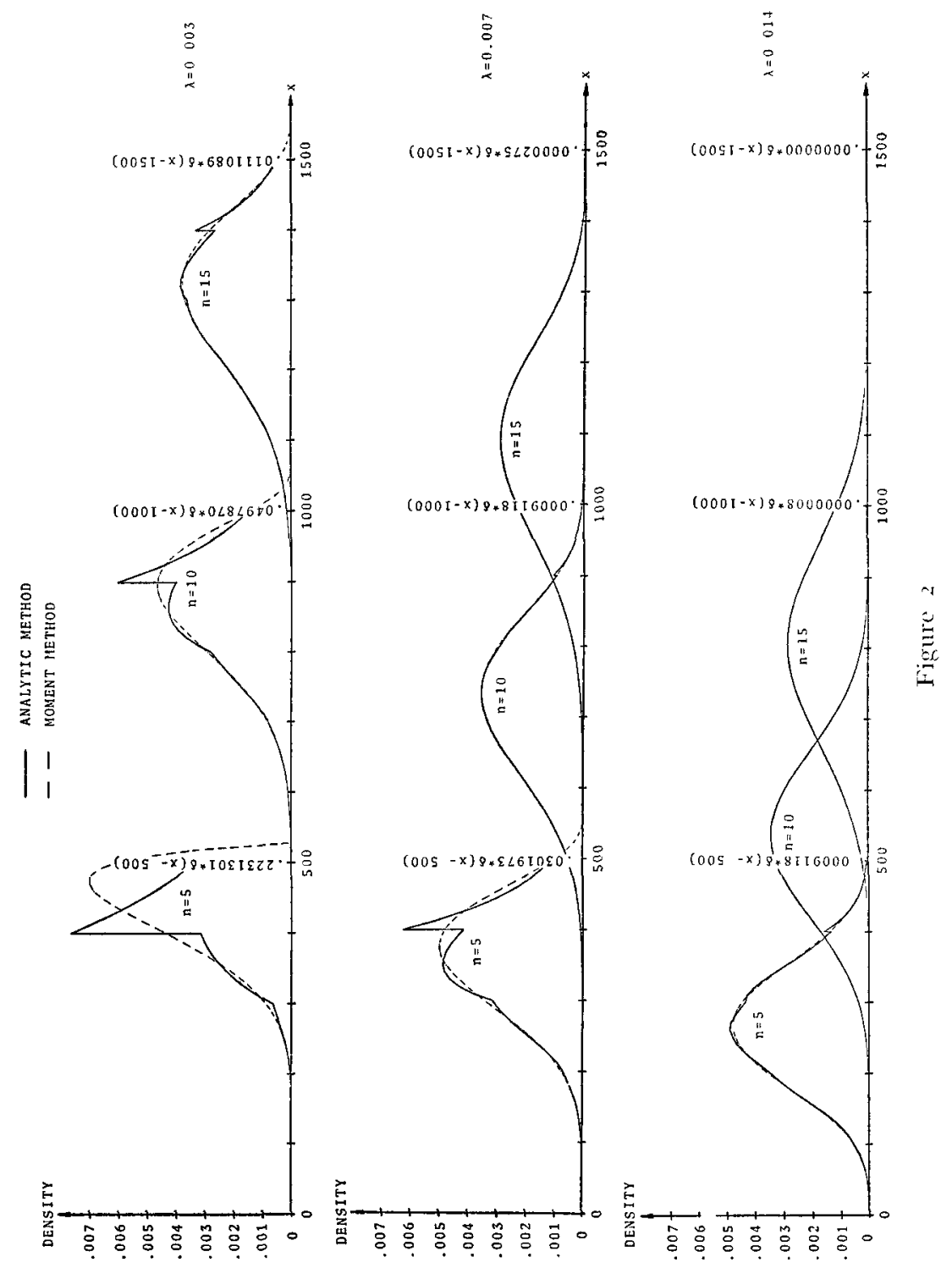




\section{Analytic versus Moment}

The comparison is based on the density functions derived by both methods to facilitate graphical representation of the results. Figure 2 illustrates the results for various values of $n$ and $\lambda$ with $D=$ I00. With the method of moments it is not possible to trace the existing discontinuities in the $n$-fold convolution of the mixed density and mass function. However, the discontinuities become less pronounced as $n$ increases, thus making the approximation more accurate $\left.{ }^{9}\right)$. Furthermore, it can be seen that the approximation, for a given $n$, improves as $\lambda$ increases. Furthermore, the mass of $D$ can be decreased by increasing $D$ thus reducing the discontinuities and improving the approximation. This observation is due to the fact that the mass at $D$ decreases as $\lambda$ increases. For problems with minor discontinuties the method of moments is clearly the more efficient procedure ${ }^{10}$ ).

\section{Conclusion}

The purpose of this paper was to describe alternative methods which can be used to derive the $n$-fold convolution of a mixed density and mass function. The methods have been applied to the mixed density and mass function given in (2). The illustrations and the comparative analysis is therefore restricted to the specific situation. The methods, however, can be used for functions other than the one given in (2) although it must be realized that the analytic method may not be applicable.

\section{REFERENCES}

[I] Abromowitz, M and I A STEgun (Edts), Handbook of Mathematical Functions, Dover Publications Inc , New York, I965

[2] Geary R C and E S Pearson, Tests of Normality, London Biometrica Office, University College, I936

[3] Feller, W, An Introduction to Probability Theory and Its Applications, Vol I and II, John Wiley \& Sons, New York, 1957 and 1966

$\left.{ }^{9}\right)$ A goodness of fit test can be applied to determine the value of $n$ above which the results of the method of moments becomes adequate for a given level of confidence

10) A comparison between the numeric and method of moments has been omitted since for a meaningful comparison a large value of $M$ would be required which in turn makes the numeric method an inefficient procedure. 
[4] Haehling von Lanzenauer, C., Optimal Insurance Purchasing Decisions, Working Paper Series No. 72, School of Business Administration, University of Western Ontario, 1972.

[5] LighthilL, M. J., Introduction to Fourier Analysis and Generalized Functions, Cambridge University Press, I958.

[6] Pearson, K., Tables of the Incomplete Beta-Function, Cambridge University Press, 1968.

[7] Seal, H., Stochastic Theory of a Risk Business, John Wiley \& Sons, New York, r969.

[8] Sмrтh, V., Optimal Insurance Coverage, Journal of Political Economy, Vol 76 , No. I, r968.

[9] Wilks, S. S., Mathematical Statistics, John Wiley \& Sons, New York, 1962 .

\section{Appendix A}

For the derivation of the third and fourth moments about the mean of a $n$-fold convolution, first consider the case of the convolution of two piece-wise continuous density functions, $g(x)$ and $h(y)$, both having finite means, $\mu_{x}$ and $\mu_{y}$, and finite variances, $\sigma_{x}^{2}=\theta^{2}(x)$ and $\sigma_{y}^{2}=\theta^{2}(y)$. Let the third moment about the mean be represented by $\theta^{3}(x)$ and $\theta^{3}(y)$ and the fourth moment about the mean be denoted by $\theta^{4}(x)$ and $\theta^{4}(y)$.

The third moment about the mean of the sum of $x$ and $y, \theta^{3}(x+y)$ can be written as

$$
\theta^{3}(x+y)=\int_{-\infty}^{\infty} \int_{-\infty}^{\infty} g(x) \cdot h(y)\left(x+y-\left(\mu_{x}+\mu_{y}\right)\right)^{3} d y d x
$$

By letting $a=x-\mu_{x}$ and $b=y-\mu_{y}$, (I 7 ) may be evaluated as follows.

$$
\begin{aligned}
\theta^{3}\left(x^{\prime}+y\right) & =\int_{-\infty}^{\infty} \int_{-\infty}^{\infty} g(x) h(y)(a+b)^{3} d y d x \\
& =\int_{-\infty}^{\infty} g(x) a^{3} \int_{-\infty}^{\infty} h(y) d y d x+3 \cdot \int_{-\infty}^{\infty} g(x) a^{2} \int_{-\infty}^{\infty} h(y) b d y d x \\
& +3 \cdot \int_{-\infty}^{\infty} h(y) b^{2} \int_{-\infty}^{\infty} g(x) a d x d y+\int_{-\infty}^{\infty} h(y) b^{3} \int_{-\infty}^{\infty} g(x) d x d y \\
& =\theta^{3}(x)+\theta^{3}(y) .
\end{aligned}
$$

Correspondingly, the fourth moment about the mean of the sum of $x$ and $y, \theta^{4}(x+y)$, can be written as 


$$
\begin{aligned}
\theta^{4}(x+y) & =\int_{-\infty}^{\infty} \int_{-\infty}^{\infty} g(x) h(y)(a+b)^{4} d y d x \\
& =\int_{-\infty}^{\infty} g(x) a^{4} \int_{-\infty}^{\infty} h(y) d y d x+4 \cdot \int_{-\infty}^{\infty} g(x) a^{3} \int_{-\infty}^{\infty} h(y) b d y d x \\
& +6 \cdot \int_{-\infty}^{\infty} g(x) a^{2} \int_{-\infty}^{\infty} h(y) b^{2} d y d x+4 \cdot \int_{-\infty}^{\infty} h(y) b^{2} \int_{-\infty}^{\infty} g(x) a d x d y \\
& +\int_{-\infty}^{\infty} h(y) b^{4} \int_{-\infty}^{\infty} g(x) d x d y \\
& =\theta^{4}(x)+6 \cdot \theta^{2}(x) \cdot \theta^{2}(y)+\theta^{2}(y) .
\end{aligned}
$$

Let $\theta_{n}^{k}$ represent the $k^{\text {th }}$ moment about the mean of the sum of $n$ random variables with a common distribution and $\theta_{1}^{k}=\theta^{k}$ denote the $k^{\text {th }}$ moment about the mean of the common distribution. Defining

$$
\begin{aligned}
\theta_{n}^{k} & =\theta^{k}(x+y) \\
\theta_{n-1}^{k} & =\theta^{k}(x) \\
\theta^{k} & =\theta^{k}(y) \quad \text { for } k=2,3, \text { and } 4
\end{aligned}
$$

and substituting into (I 8 ) leads to

$$
\theta_{n}^{3}=\theta_{n-1}^{3}+\theta^{3} \text {. }
$$

From (20) it follows that

$$
\theta_{n}^{3}=n \theta^{3}
$$

Correspondingly, (I9) becomes

$$
\theta_{n}^{4}=\theta_{n-1}^{4}+6 \theta_{n-1}^{2} \theta^{2}+\theta^{4}
$$

From (22) it follows that

$$
\theta_{n}^{4}=n \theta^{4}+3\left(n^{2}-n\right)\left(\theta^{2}\right)^{2} .
$$

